\title{
Simple Walking Strategies for Hydraulically Driven Quadruped Robot over Uneven Terrain
}

\author{
Jungsan Cho*, Jin Tak Kim*, Jungyoung Kim**, Sangdeok Park* and Kab Il Kim ${ }^{\dagger}$ \\ Abstract - Jinpoong is a quadruped walking robot developed by the Korea Institute of Industrial \\ Technology. Jinpoong was developed for use in surveillance, reconnaissance, transportation, and \\ guidance tasks in atypical environments such as those encountered after a disaster. To perform these \\ tasks successfully, the robot must be able to walk stably over uneven terrain. The aim of this study was \\ to develop a method for absorbing the impacts experienced by the robot while walking and a method \\ for resisting external forces applied to the side (sagittal plane) of the robot so that it can walk stably. \\ We demonstrate the efficacy of the methods developed through experiments.
}

Keywords: Jinpoong, Walking robot, Hydraulic quadruped robot, Balance control

\section{Introduction}

The most promising aspect of quadruped robots is that they can easily adapt to and walk over uneven terrain, such as that found in mountainous areas, owing to their structural similarity to quadruped animals. This advantage can be leveraged for applications such as entering collapsed structures and providing military transportation. To accomplish such tasks, the range over which a quadruped robot can operate must be expanded to include uneven terrain, such as that found in mountainous areas, and stability in walking on uneven terrain must be achieved.

Dynamic locomotion of quadruped robots has been investigated using a variety of approaches [1]. However, several major problems associated with dynamic locomotion of quadruped robots need to be resolved. One of the major problems is the need to design mechanical systems that are able to deliver the energy needed to sustain dynamic locomotion during intermittent periods of foot contact. When this need is considered together with the needs for acceptable energy efficiency and an acceptable weight, the demands placed on the actuation systems of a quadruped robot are difficult to fulfill.

Stabilization and control of dynamic locomotion are also significant problems. Successful control strategies are based on the observations of Raibert [2], who demonstrated the importance of two essential ingredients: control of the body through the hip stance and foot placement control for balance recovery.

The driving system consists of electric motors and hydraulic motors. Significant examples of quadruped

$\dagger \quad$ Corresponding Author: Dept. of Electrical Engineering, Myong Ji University, Korea. (kk1@mju.ac.kr)

* Robotics R\&D Group, Korea Institute of Industrial Technology, Korea. (\{chojs, jintagi, sdpark\}@kitech.re.kr)

** Dept. of Intelligent Robot Engineering, University of Science \& Technology, Korea. (kjy880527@kitech.re.kr)

Received: November 27, 2014; Accepted: March 7, 2016 robots with electric motors are the robot developed by Hirose et al. [3], Sony's AIBO [4], and Little Dog [5]. In combination with high position gain control and kinematic planning, these robots demonstrated statically and quasistatically stable walking gaits. One approach to achieving stable walking is to use springs in combination with electric motors. Examples of this approach are the quadruped robots Tekken [6], KOLT [7], and Cheetah [8]. These quadruped robots are able to perform bounding and trotting on flat ground.

Significant examples of quadruped robots with hydraulic actuators are BigDog [9] and HyQ [10]. Boston Dynamics has demonstrated an advanced level of performance on uneven terrain with their BigDog, AlphaDog, and Wild Cat robots. BigDog was stabilized by applying torque to the hip joint during the stance phase. The torque applied was computed as a function of the body pitch and roll angles and rates by a proportional-derivative (PD) controller. The Italian Institute of Technology (IIT) has demonstrated the practicality of a hydraulic robot with their HyQ design.

HyQ utilizes a floating-base inverse dynamics-based feed-forward servo control algorithm to ensure that position tracking is achieved with low PD control gains. All of these robots operate using a walking stabilization algorithm that is based on the application of torque to control the joints. While this type of algorithm is quite useful, we think that a better approach would be one that combines balance control with force control.

Hydraulic actuation offers the advantages of substantial power, a wide range of speed, small size, and low weight [11]. Because of these advantages, hydraulic actuation is often used in robots that lift heavy loads. Jinpoong (shown in Fig. 1) is a hydraulically operated quadruped robot that is powered by an engine. Jinpoong was developed for military transportation applications [12-14].

This study was conducted to develop a method for ensuring the stability of a hydraulic robot system as it 


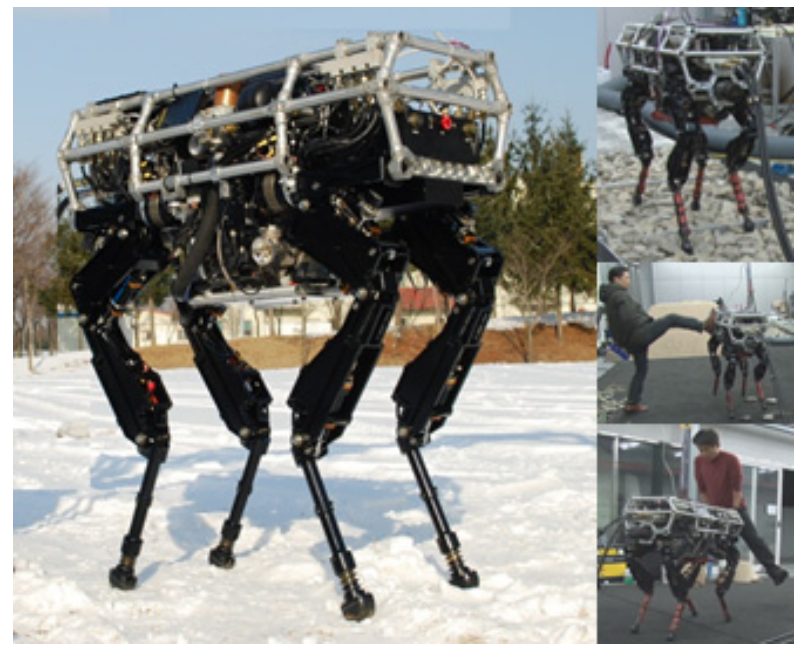

Fig. 1. Jinpoong, a hydraulically actuated quadruped robot

walks over uneven terrain, using a controller that operates based on joint position control. In this paper, we present the results of experiments conducted to verify the efficacy of the method developed. We developed a hydraulically actuated quadruped robot as shown in Fig. 1 which experiments are walking on the gravelly field, kicked from the side, and mounted by person. In Section 2, we describe the configuration of Jinpoong, and in Section 3, we explain the walking strategy. The verification tests conducted and the results obtained are described in Section 4.

\section{Hardware Specifications}

Jinpoong uses hydraulic connections to power its components when walking. These components include the body frame, the hydraulic system, the power system, and the control system. The body frame was designed to protect the internal components. A component arrangement was designed to ensure stability during walking. A separate structure that could be mounted on the engine through the lower body was attached to the inner body frame so that the components did not protrude outside the body frame. The walking stability of the robot was improved by lowering the center of gravity.

The hydraulic system drives the hydraulic pump that powers the engine and generates and supplies the oil pressure required to drive the joint actuator. In view of the arrangement of the component modules of the robot, such as the leg modules, controller and sensors, engine, and muffler, the hydraulic system was designed together with the body structure. To ensure smooth movement during walking and to optimize the space efficiency of the hydraulic components between the hydraulic pumps and manifolds, such as storage tanks, the trajectory and radius of curvature of the hydraulic hose were taken into consideration in the design of the hydraulic system. The hydraulic system was designed to supply hydraulic fluid to

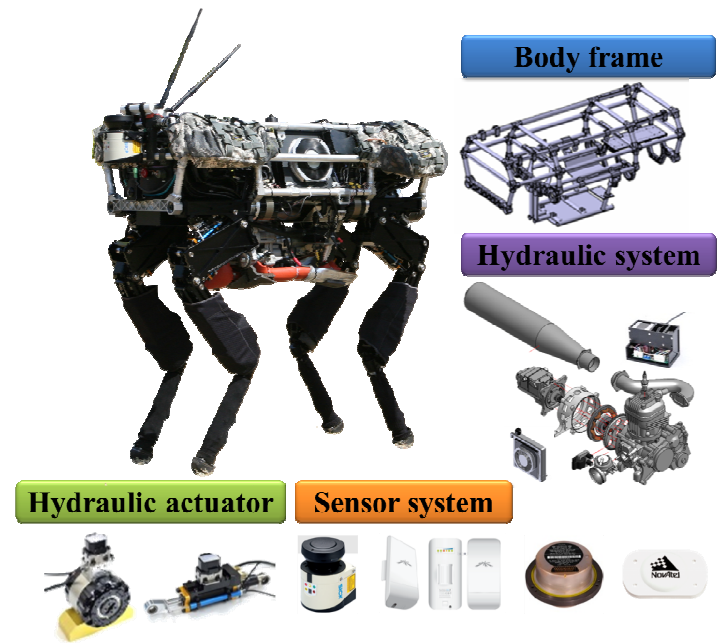

Fig. 2. Composition of Jinpoong

the joint actuator located within each of the four legs by means of four sub-manifold folds.

Jinpoong has onboard systems that provide power, actuation, sensing, controls and communications, as shown in Fig. 2. Jinpoong uses a two-stroke, 30-horsepower engine and a swash-plate-type hydraulic pump to provide power for walking across open terrain. The maximum hydraulic pressure is 210 bars, and the maximum flow is $35 \mathrm{lpm}$. Jinpoong has 16 degrees of freedom (each of the four legs having four degrees of freedom) for walking over uneven terrain. The height of the robot is $1.2 \mathrm{~m}$, the length is $1.1 \mathrm{~m}$, the width is $0.4 \mathrm{~m}$, and the weight is $120 \mathrm{~kg}$. These dimensions were selected on the basis of a typical human being's height, mobility, speed, and load-carrying capacity.

The legs consist of rolling joints for the hips, with rotary hydraulic actuators, and pitching joints for the hips, knees, and feet, with linear hydraulic actuators. The operating range of the rotational actuator is $\pm 70^{\circ}$, and its output power is $400 \mathrm{Nm}$ at a pressure of 210 bars. The operating range of the linear actuator is $54 \mathrm{~mm}$, and its output power is $8000 \mathrm{Nm}$ at 210 bars. The actuators are controlled by electronic servo-valves (Star Hydraulics Ltd., UK). All of the actuators are equipped with potentiometers to measure their positions.

The linear hydraulic actuators are lighter and yet more powerful than rotary actuators and were designed to move quickly despite the robot carrying a payload over rough terrain. The hydraulic actuators are placed on the upper parts of the legs to make the lower parts lighter.

This design allows the overall center of gravity to be closer to the center of the robot, thus improving stability and making the robot less susceptible to the dynamics of a swinging leg. Rotary actuators rather than linear actuators are used for the hip joint to optimize the size and shape of the platform. The modular design concept employed for the components such as the controllers also helped to optimize the size and shape of the platform. 
An inertial measurement unit (HG1700, Honeywell Company, USA) is used to measure body posture and inertia. The control system consists of a power control unit (PCU) to control the engine and hydraulic power, a leg control unit (LCU) to control the action of each joint and leg, a sensor processing unit (SPU) to collect information necessary for walking and navigating, and a behavior control unit (BCU) to control walking and other actions. These units are connected together through an EtherCAT network with a sampling time of $1 \mathrm{~ms}$.

\section{Walking Strategies}

Jinpoong was developed to be able to move over uneven terrain while achieving a high level of dynamic performance in tasks such as carrying a heavy load. Compliance of the foot-end and posture control in the sagittal and lateral planes are needed to maintain the steady dynamic motion of a quadruped walking robot. This study was conducted to develop a method for achieving stable trotting by a quadruped walking robot. The method developed relies on position-based z-axis compliance control using a spring mechanism and posture control for the sagittal and lateral planes that uses an inertial measurement unit (IMU) sensor.

Hydraulic actuators are inherently velocity sources: electrical signals regulate their velocity responses. Such systems are therefore by nature high-impedance (mechanically stiff) systems. Theoretically, the output force of a hydraulic actuator is infinity at zero input $[15,16]$. Force control can be implemented by installing torque sensors to control the torque of the joints. However, for legged robots, force control using hydraulic actuators is an inherently difficult problem. Problems such as the limited durability of torque sensors and the limits on the feedback control make it difficult to absorb instantaneous impact. Research is needed to examine the applicability of force control to walking on uneven terrain. We propose a strategy for walking of a hydraulically driven quadruped robot over uneven terrain that uses a simple method based on relatively easy position control of hydraulic actuators.

\subsection{Position-based Z-axis compliance control}

When a walking robot traverses uneven terrain in a given direction, which we consider here to be the direction of the z-axis, the reaction force of the ground on the robot's foot end is delivered to each joint, which adversely affects the walking control performance and the durability of the leg module. The direction of $\mathrm{Z}$-axis is vertical to the ground. If the joint gain is increased to improve position accuracy, the robot becomes very stiff, which increases the force on each joint when the robot is traversing uneven terrain. It is difficult to attain compliance using a hydraulic actuator even if position control gain is reduced. An

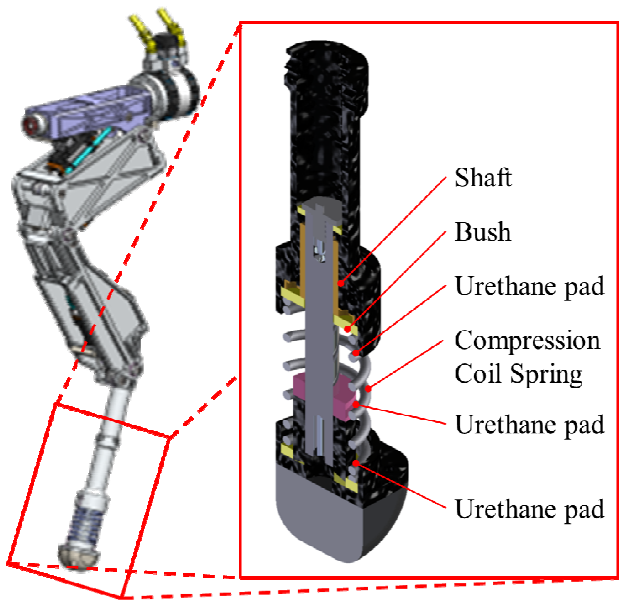

Fig. 3. Design of foot mechanism

electronic motor can achieve compliance through PD control [17], but it is difficult to do so using a hydraulic actuator. Studies have shown that a torque/force sensor can be used to control the torque of the joint and thus achieve compliance $[9,10]$. In this study, we considered the hydraulic joint to be a precision positioning control source and installed a spring at the foot end as a shock absorber. However, if a mechanical spring is installed, the stiffness cannot be varied depending on the situation, which could adversely affect walking stability. To overcome this problem, we designed a dual-stiffness shock absorber capable of absorbing the initial impact upon landing and then stiffening in the stance phase, as shown in Fig. 3.

Trotting, one of Jinpoong's walking patterns, is a periodic pattern in which the diagonally opposite legs move simultaneously. The trotting pattern is determined by the desired position trajectories for the foot end of each leg. The reference trajectories for the joint position can be calculated by the application of inverse kinematics [18]. A closed-form solution for the inverse kinematics of this system, considering balance control, was obtained. The foot contact states and desired position trajectories of the foot end along the z-axis, considering balance of the body, can be calculated using the following formulas.

$$
\begin{gathered}
z_{d, s w}=\left(h_{\max }-z_{d, 0}\right) \sin \left(\omega_{s w} t\right) \quad \text { when } t<T_{s w} / 2 \\
z_{d, s w}=z_{d, s w}-\left(\frac{h_{1}}{T_{s w}-T_{2}}\right) t_{s} \quad \text { when } t<t_{\text {touch }} \\
z_{d, s t}(\text { front })=z_{n}(\text { front }) \\
+\left(\frac{z_{n}(\text { front })-z_{n}(\text { hind })}{2}\right) t_{s} \\
z_{d, s t}(\text { hind })=z_{n}(\text { hind }) \quad t_{s} \text { when } t<T_{\text {st }} / 2 \\
+\left(\frac{z_{n}(\text { hind })-z_{n}(\text { front })}{2}\right) \\
z_{d, s t}=z_{n}+l_{w} t_{s} \sin \theta \quad \text { when } t<t_{\text {touch }}
\end{gathered}
$$




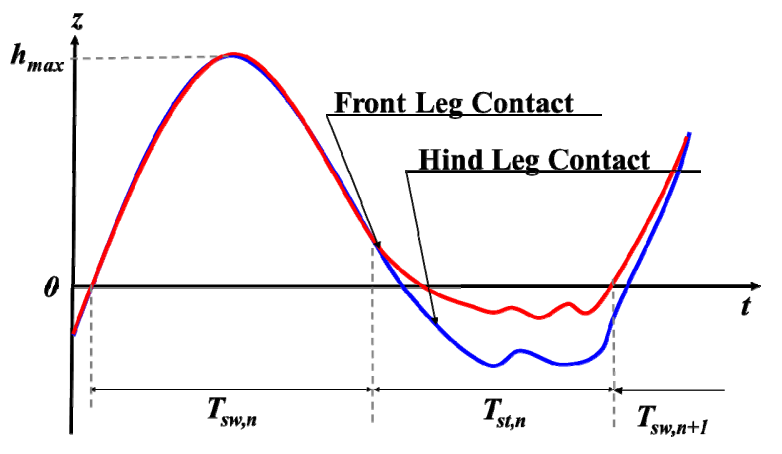

Fig. 4. Example of foot trajectory along z-axis

where $z_{d, s w}$ and $z_{d, s t}$ are the desired positions of the foot end of each leg on the z-axis in the swing and stance phases, respectively; $\omega_{s w}$ is the walking period in the swing phase. $z_{n}$ is the real position of the end effector of each leg on the $\mathrm{z}$-axis; $z_{d, 0}$ is the leg's position on the z-axis at the start of the swing; (front) and (hind) are the front and hind legs, respectively; $T_{s w}$ and $T_{s t}$ are the gait cycle time of the swing and stance phases, respectively; $t_{s}$ is the sampling interval; ( $1 \mathrm{~ms}$ ); $t_{\text {touch }}$ is the contact time of one of the swinging legs; $h_{l}$ is the height from the predicted ground position to the current position; $h_{\max }$ and $T_{2}$ are the maximum foot-end height and swing-down time, respectively; $l_{w}$ is the length of the stance leg, and $\theta$ is the angle of the body tilt as measured by the IMU sensor.

As Fig. 4 shows, the z-axis trajectory of the foot end of each leg can be modified by the ground contact state or the body posture. On uneven terrain, the contact time and leg length should be controlled according to the state of the ground or the body. Eqs. (1) and (2) can be used to generate the z-axis trajectory of the foot end during the swing phase and landing-to-stance phase, respectively. To enhance the locomotion stability, it is suggested that the length of the leg be controlled using the IMU sensor during the stance phase. The contact state is determined by the potentiometer connected to the shaft installed in the shock absorber, as shown in Fig. 3. The trotting cycle is divided into four phases: swing up, swing down, landing, and stance. Each phase prescribes a set of sensor conditions that trigger the transition to the next phase and a set of control actions to be applied in that phase. The phase transitions track the behavior of the machine and synchronize the functions controlling the vertical thrust, attitude, and foot-end placement in response to the ongoing behavior.

\subsection{Balance control}

To allow the robot to walk on uneven terrain, balance control is required for maintaining posture in the event of a disturbance. Physical disturbances that could affect the robot can be divided into three categories. First, the robot body could fall after being acted upon by an external force, as shown in Fig. 5(a). Second, when the robot is trotting on

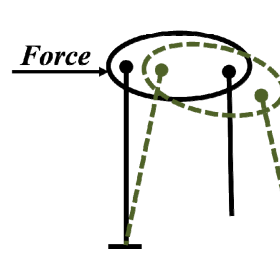

(a)

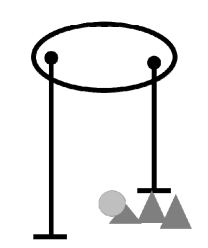

(b)

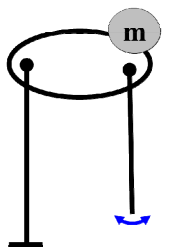

(c)
Fig. 5. Three types of disturbances that can act on the body of the robot: (a) An external force on the side of the robot, (B) Walking on uneven terrain, (c) The payload located to one side of the robot's center of gravity

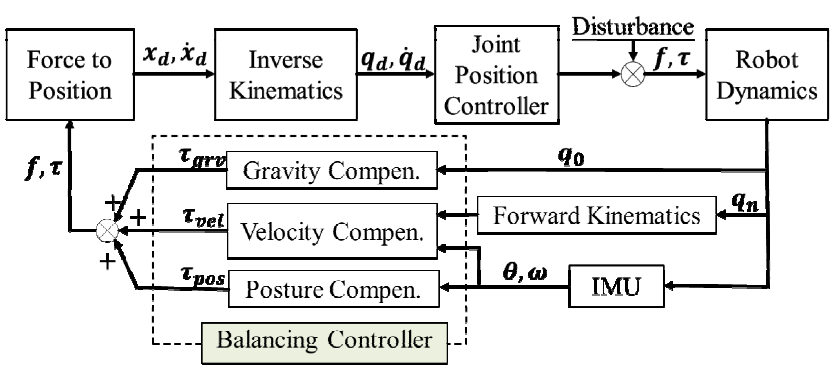

Fig. 6. Block diagram of balance control for stance legs

uneven terrain, its leg-landing height could change, or its foot end could suddenly slide on the ground, as shown in Fig. 5(b). Third, the payload could be located to one side of the robot's center of gravity, as shown in Fig. 5(c). Other possible disturbances include joint position sensor errors, IMU sensor errors, and distortion of the robot frame. From these cases, the output data from the IMU sensor installed in the robot is similar so we can recognize the dynamics which effects to the body of the robot is similar. To address such situations, we designed the robot so that it can obtain feedback on the orientation and angular velocity of the body from the body posture sensor and maintain a constant body attitude. We calculate a target value for the foot end using a simple dynamics model.

The trotting of a quadruped robot involves the periodic repetition of the phases from stance to swing. Therefore, a controller is required for each phase. First, we consider the controller for the stance leg. Balance control for the stance legs consists of attitude compensation in accordance with the body tilt, gravity compensation in accordance with the angle of the hip joint, and velocity compensation in accordance with the body velocity. A block diagram of the balance control for stance legs is shown in Fig. 6 .

Attitude compensation means that the body tilts to remain horizontal by generating a positive torque in accordance with the angle of the body tilt, as shown in Fig. 7(a). Raibert presented the following equation for calculating the torque from the angle of tilt [2]:

$$
\tau_{p o s}=-\lambda_{p}\left(\theta_{d}-\theta\right)+\lambda_{d} \omega
$$




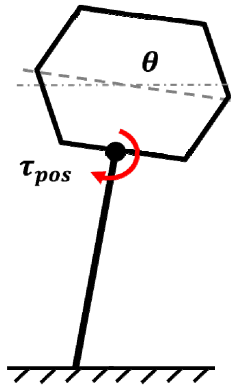

(a)

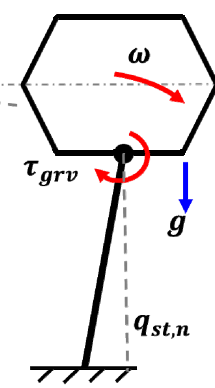

(b)

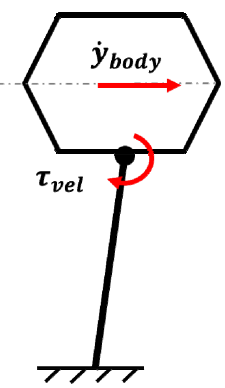

(c)
Fig. 7. Balancing control in the sagittal plane during the stance phase: (a) Control of the body tilt; (b) Control of the body velocity; (c) Gravity compensation of the resulting joint angle

where $\tau_{p o s}$ is the torque required for attitude compensation; $\lambda_{p}$ and $\lambda_{d}$ are the coefficients of the body tilt and angular velocity, respectively; $\theta$ and $\omega$ are the angle and angular velocity of the body tilt, respectively, as measured by the IMU sensor; and $\theta_{d}$ is the desired angle of the body tilt.

According to a simple inverted pendulum model, if the hip joint angle, $q_{s t, n}$, is not zero in the state in which the body velocity in the previous phase was approximately zero, then the robot will incline to one side under the influence of gravity, as shown in Fig. 7(b). We use the following equation to calculate the torque required for gravity compensation:

$$
\tau_{g r v}=m g l \sin \left(q_{s t, n}\right)
$$

where $\tau_{g r v}$ is the torque required for gravity compensation, $m$ is the mass of the robot. $g$ is the acceleration due to gravity, $l$ is the leg length, and $q_{s t, n}$ is the current hip joint angle during the stance phase.

If the body is controlled only by Eq. (3), the hip joint stops rotating when the angle of the body tilt is zero, and the robot is more likely to fall because of the acceleration in the forward direction. This means that the velocity of the robot body must be maintained in proportion to the velocity in the previous phase, as shown in Fig. 7(c). The torque required for velocity compensation can be calculated from the following equation:

$$
\tau_{v e l}=\lambda_{v} \hat{\dot{y}}_{b o d y}
$$

where $\tau_{\text {vel }}$ is the torque required for velocity compensation, $\lambda_{v}$ is a coefficient of proportionality that can be determined experimentally, and $\dot{y}_{b o d y}$ is the body velocity with respect to the $y$-axis. The y-axis body velocity can be estimated using kinematic information and the IMU output.

The desired torque is applied to the joint by the joint position controller. The joint torque can be calculated by modifying the desired value. The hip joints determine the movement of the robot in the $y$-axis direction. Therefore,

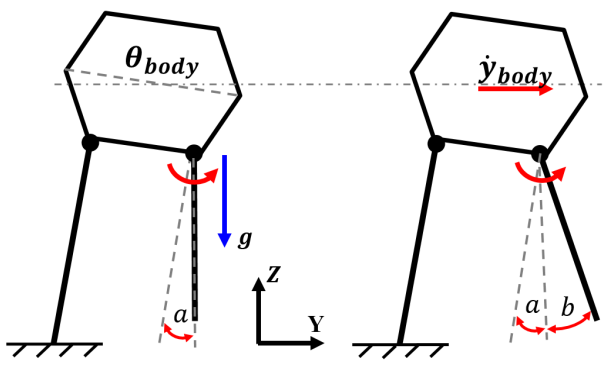

(a)

(b)

Fig. 8. Balance control in the sagittal plane during the swing phase: (a) The desired position of the foot end depending on the body tilt, (b) The variation in the foot-end position depending on the body velocity

the desired change in the hip joint position can be determined from the output of the balance controller. The balance control equation is as follows:

$$
\begin{aligned}
\Delta q_{d}=-\lambda_{p}\left(\theta_{d}-\theta\right) & +\lambda_{d} \omega+\operatorname{mgl} \sin \left(q_{s t, 0}\right)+\lambda_{v} \hat{\dot{y}}_{b o d y} \\
\tau_{j o \text { int }}= & K_{p}\left(q_{s t, n}+\Delta q\right)
\end{aligned}
$$

where $q_{d}$ is the desired joint position determined from the output of the balance controller, $\tau_{\text {joint }}$ is the joint torque produced by the $\Delta q_{d}$ value, and $K_{p}$ is the gain of the joint position controller. The coefficients of the balance control equation are obtained using a heuristic approach.

The controller for the swinging leg determines the position of the foot end in the y-direction, depending on the body slope and the current body velocity, as shown in Fig. 8. In the swing phase as shown in Fig. 8(a), the hip joint moves as the direction of the foot-end of the swinging leg keeping parallel with the direction of gravity. In addition, the landing point of the leg is calculated based on the current body speed as shown in Fig. 8(b). The controller determines the foot placement of the swinging leg according to the following formula:

$$
y_{s w, d}=\zeta_{1} L \sin \theta+\zeta_{2} \int \dot{y}_{b o d y}
$$

where $y_{s w, d}$ is the desired position of the swinging leg on the y-axis; $L$ is the leg length; and $\zeta_{1}$ and $\zeta_{2}$ are the coefficients of the body inclination and the body speed, respectively, in the swing phase.

\section{Experimental Results}

This section describes the tests that we performed to verify the two proposed simple strategies and the results that we obtained. First, we performed tests of the positionbased z-axis compliance control. We conducted a marching in place experiment to analyze the characteristic of the motion in Z-axis. For the experiment, we did not input any 


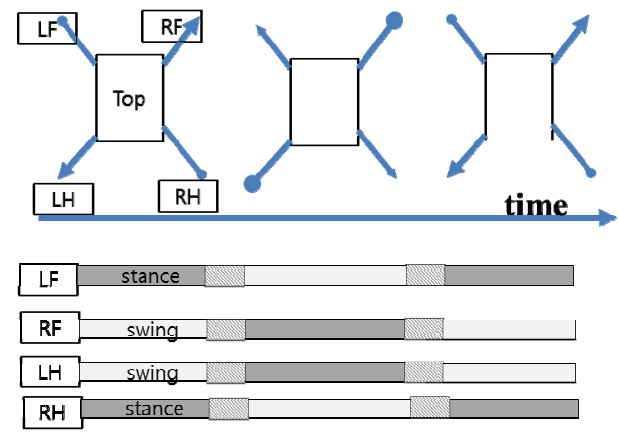

(a) Trotting sequence

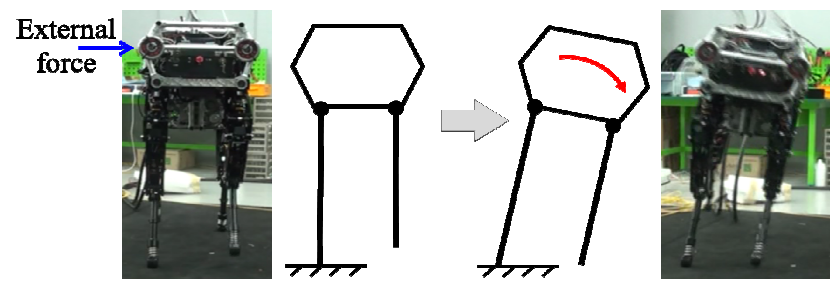

(b) Balance control for stance leg

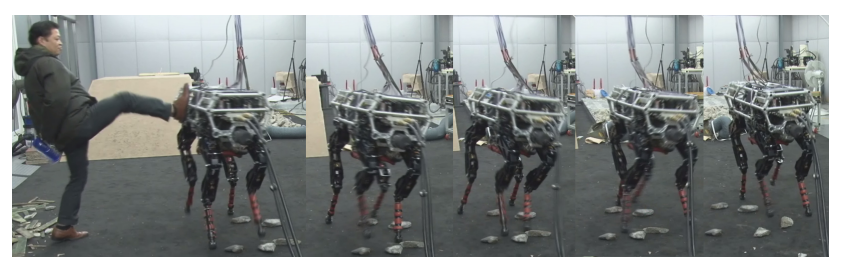

(c) External force test: kicking the robot

Fig. 9. Balance and external force tests

commands about X- and Y-axis. The trot gait was chosen as the dynamic gait. In the trot gait, the diagonally opposite limbs move in unison, as shown in Fig. 9(a). The gait cycle changes from the swing phase to the stance phase every 0.4 $\mathrm{s}$; however, as described earlier, the timing of the foot-end changes depends on the ground contact.

Figure 10(a) shows the body's Z-axis acceleration, as measured by the IMU mounted on the body, according to each shock absorber state. The IMU sensor measures both the acceleration of the body and the gravity. Therefore the upper graph in Fig. 10(a) is measured including offset of the gravity acceleration $9.81 \mathrm{~N}$. If the robot body is inclined or walking on the uneven terrain, the contact time of each leg and the leg length are changed. The ground state is estimated by the body posture, the ground contact states, and the current leg length. As lower graph in Figure 10 (a) shows, the foot-end trajectory in the Z-axis direction is modified by Eq. (1), (2) and the ground contact state, and the body acceleration in the Z-axis direction is reduced by the shock absorber and controller.

Tests of the proposed balance control method (Eq. (7)) were also conducted. We performed tests of the control of the stance leg when two of the robot's diagonally opposite legs were raised, as shown in Fig. 9(b). In Fig 10(b) and (c),

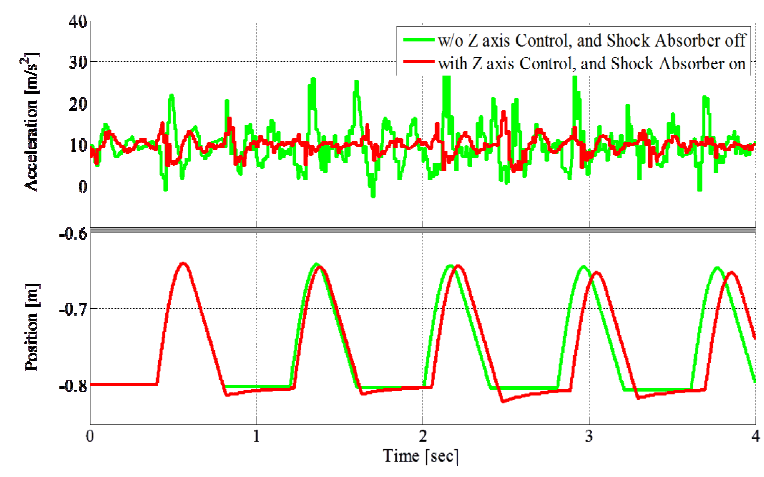

(a) Shock absorber test

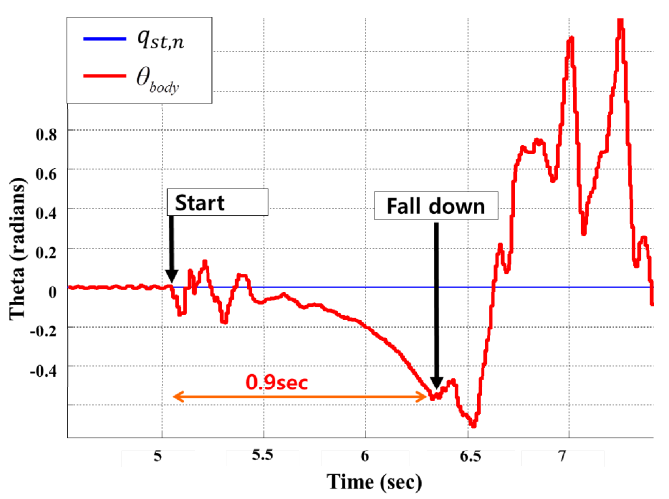

(b) Balance control for stance leg: uncontrolled

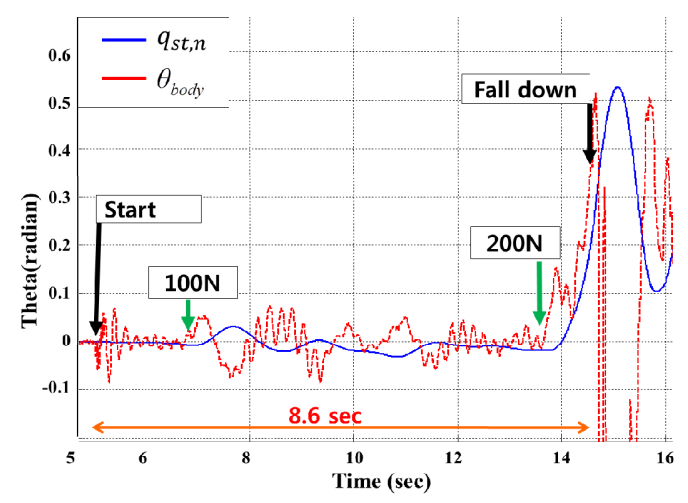

(c) Balance control for stance leg: controlled

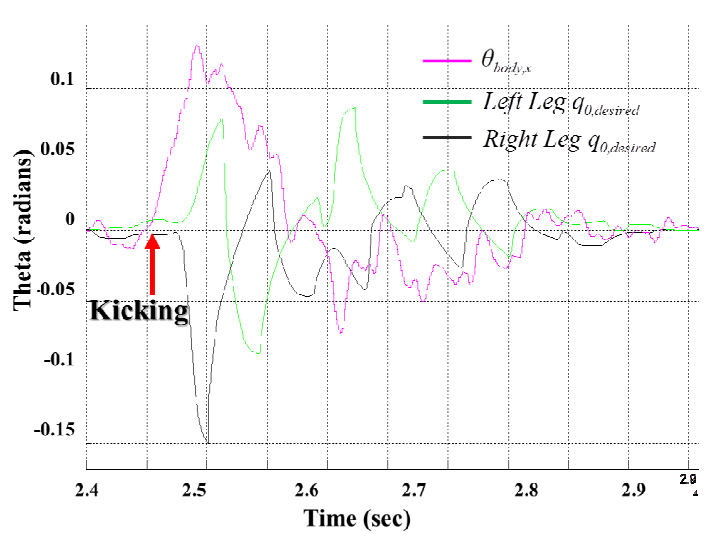

(d) Kick test

Fig. 10. Results of balance and external force tests 
the start indication means when the robot lift both legs on the diagonal. The body shakes when the body dynamics changed. As Fig. 10(b) shows, without balance control, the robot fell in less than $1 \mathrm{~s}$, even when no external force was applied. We can seem that the body shuddered to a halt, as shown in Fig. 10(c). The stable state is the body tilt angle in a section converging $\pm 0.02 \mathrm{rad}$. The body tilt angle converged to the section when an external force of $100 \mathrm{~N}$ is applied. These results show that the method of balance control for the stance leg proposed in this study maintains the body tilt angle at nearly zero degree for $8.6 \mathrm{~s}$, even when a sideways external force acts on the robot. The body fell when an external force of $200 \mathrm{~N}$ is applied, after the body tilt angle converged the section.

As Fig. 9(c) shows, the walking stability (controlled using Eqs. (7) and (8)) was tested by applying a force of approximately $400 \mathrm{~N}$ from the side by kicking the robot.

When this external force was applied, the hip joints of the left and right legs moved as predicted, the body tilt decreased, and the robot returned to a steady state, as shown in Fig. 10(d).

\section{Conclusions}

This paper describes a strategy for maintaining the stability of a position-based hydraulic robot system while walking over uneven terrain. To achieve stability while walking, we developed a method for achieving stable trotting by a walking quadruped robot. The method relies on position-based z-axis compliance control using a spring mechanism and posture control in the sagittal and lateral planes using an IMU sensor. The proposed strategies were verified through tests in which the foot-module shock absorber was operated during a free fall and in which a sideways force was applied while the robot was trotting. The results of these tests show that the proposed strategies enable the robot to walk steadily over uneven terrain.

\section{Acknowledgments}

This work was supported by the Industrial Strategic technology development, 10052968, Development of core components and systems for special purposed machine in disaster environment funded By the Ministry of Trade, Industry \& Energy (MI, Korea)

\section{References}

[1] L.J.R. Yibin, L. Bin, and R. Xuewen, "Research of mammal bionic quadruped robots: a review," in Proc. IEEE International Conference on Robotics, Automation and Mechatronics, 2011, pp. 166-171.

[2] M. Raibert, Legged robots that balance, Cambridge,
MA, MIT, 1986.

[3] S. Hirose, Y. Fukuda, K. Yoneda, A. Nagakubo, H. Tsukagoshi, K. Arikawa, G. Endo, T. Doi, and R. Hodoshima, "Quadruped Walking Robots at Tokyo Institute of Technology," IEEE Robotics and Automation Magazine, 2009, vol. 16, no. 2, pp. 104-114.

[4] M. Fujita and H. Kitano, "Development of an autonomous quadruped robot for robot entertainment," Autonomous Robots, 1998, vol. 5, pp. 7-18.

[5] M. Kalakrishnan, J. Buchli, P. Pastor, M. Mistry, and S. Schaal, "Learning, planning, and control for quadruped locomotion over challenging terrain," Int. J. of Robotics Research, 2010, vol. 30, no. 2, pp. 236-258.

[6] H. Kimura, Y. Fukuoka, and A.H. Cohen, "Adaptive dynamic walking of a quadruped robot on natural ground based on biological concepts," Int. J. of Robotics Research, 2010, vol. 26, no. 5, pp. 475-490.

[7] J.G. Nichol, S.P.N. Singh, K.J. Waldron, L.R. Palmer, and D.E. Orin, "System Design of a Quadrupedal Galloping Machine," Int. J. of Robotics Research, 2004, vol. 23, no. 10-11, pp. 1013-1027.

[8] S. Seok, A. Wang, D. Otten, and S. Kim, "Actuator Design for High Force Proprioceptive Control in Fast Legged Locomotion," in Proc. IEEE International Conference on Intelligent Robots and Systems, 2012, pp. 1970-1975.

[9] M. Raibert, K. Blankespoor, G. Nelson, and R. Playter, "Bigdog, the rough-terrain quadruped robot," in Proc. International Federation of Automatic Control, 2008, pp. 10822-10825.

[10] T. Boaventura, C. Semini, J. Buchli, M. Frigerio, M. Focchi, and D. G. Caldwell, "Dynamic torque control of a hydraulic quadruped robot," in Proc. IEEE International Conference on Robotics and Automation, 2012, pp. 1889-1894.

[11] H.E. Merritt, "Hydraulic Control Systems," New York: Wiley, 1967.

[12] B. So, T. Kim, O. Kwon, S. Park and W. Son, "Development of quadruped walking robot using a hydraulic rotary actuator," in Proc. of IEEE International Symposium on Robotics, 2008, pp. 314-318.

[13] S.R. Lee, J.S. Cho, and S. Park, "Position based Impedance Control of a Hydraulic Actuator for a Walking Robot," in Proc. International Conference on Climbing and Walking Robots and Support Technologies for Mobile Machines, 2012, pp. 781-788.

[14] T. Kim, S. Park, and B.J. Yi, "A Composite Algorithm for Flow Rate Reduction and Stable Body Trajectory Generation in a Hydraulic Actuated Quadruped Robot with Kinematic Redundancy," in Proc. IEEE International Symposium on Mechatronics and Automation, 2011, pp. 343-348.

[15] M. V. Sivaselvan, A. M. Reinhorn, X. Shao, and S. Weinreber, "Dynamic Force Control with Hydraulic Actuators Using Added Compliance and Displace- 
ment Compensation," Earthquake Engineering and Structural Dynamics, vol. 37, no. 15, 2008, pp. 17851800.

[16] C. L. Nachtigal, and M. D. Martin "Instrumentation and control: fundamentals and applications," John Wiley and Sons, New York, 1990.

[17] D.T. Tran, I.M. Koo, H. Moon, J.S. Cho, S. Park, and H.R. Choi, "Motion control of a quadruped robot in unknown rough terrains using 3D spring damper leg model," in Proc. of International Conference on Robotics and Automation, 2012, pp. 4931-4936.

[18] J. T. Kim, J.S. Cho, B. Y. Park, and S. Park, "Inverse kinematics for control of Jinpoong," in Proc. of International Conference on Ubiquitous Robots and Ambient Intelligence, 2013, pp. 470-472.

[19] J. Cho, J.T. Kim, S. Park, Y. Lee, and K. Kim, "Jinpoong, posture control for the external force," in Proc. IEEE International Symposium on Robotics, 2013, pp. 1-2.

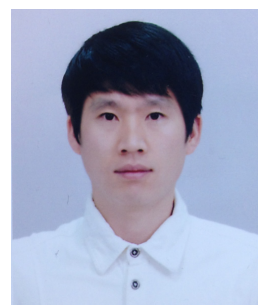

Jungsan Cho He received B.S. and M.S. degree in electrical engineering from Kumoh National Institute of Technology, Korea in 2002 and 2004 respectively. He received his Ph.D. in Electronical Engineering form Myong Ji University, in 2015. Since 2011, he has been with the Department of Robotics, Korea Institute of Industrial Technology, Korea, where he is currently a Researcher. His research interests include the areas of Hydraulic Robotics, especially in Quadruped, Manipulation and Hydraulic Control.

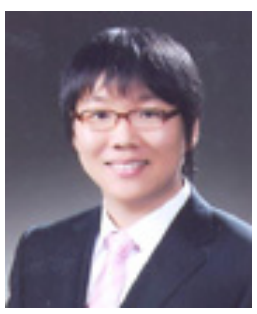

Jin Tak Kim He received B.S. in mechanical engineering from Hannam University, Korea in 2008, and M.S. from Hanyang University, Korea in 2011. He is currently a research fellow in Robotics group at Korea Institute of Industrial Technology, Korea. His research interests include the areas of Hydraulic Robotics, especially in Manipulator, Manipulation and Hydraulic Control.

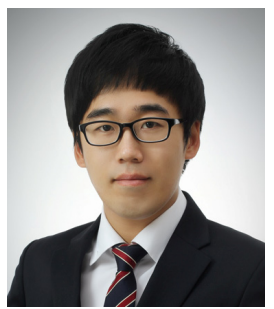

Jungyeong Kim $\mathrm{He}$ received the B.Eng. degree in mechanical engineering from Konkuk University, Korea in 2012. He is a M.Eng. degree candidate in intellegent robot engineering at University of Science \& Technology and also a researcher fellow in Department of Robotics, Korea Institute of Industrial Technology. His current interests include the areas of hydraulic robotics, espectially in actuator design and control.

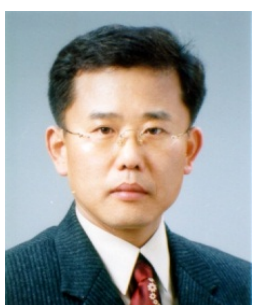

Sangdeok Park received his B.S. degree in Mechanical Design at Yeungnam University in 1998 and M.S. and Ph.D. degrees from the department of Mechanical Engineering at Pohang University of Science and Technology (POSTECH) in 1990 and 2000, respectively. Currently he is a principal researcher of Robotics R\&D group of KITECH. His research interests include the design and control of quadruped walking robots, wearable robots and hydrauliccally driven robots.

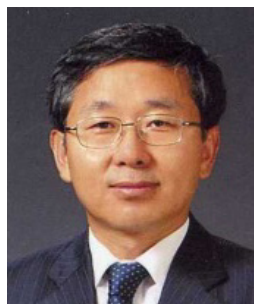

Kab Il Kim graduated Seoul National University (SNU) for B.S. in 1979, Korea Advanced Institute of Science and Technology (KAIST) for M.S. in 1981, and Clemson University in South Carolina (USA) for Ph.D. in 1990, respectively. Since 1991, he has been with the Department of Electrical Engineering, Myongji University, Korea, where he is currently a Professor. He studied Two Arm Coordination, Load Distribution, and Humanoid Robotics as a main research topic. On the practical aspect, he is currently interested in the area of Field Robotics and Military robotics. 